\title{
Spatial Cross-Correlation of Multilink Propagation Channels in Amplify-and-Forward Relay Systems
}

\author{
Xuefeng Yin \\ School of Electronics and Information Engineering, Tongji University, Shanghai, China \\ Email: yinxuefeng@tongji.edu.cn
}

\begin{abstract}
In this contribution, an analytical expression is derived for the correlation matrix of the multiple-input multiple-output (MIMO) propagation channel for the amplify-and-forward relay transmission systems. In order to understand the crosscorrelation mechanism, we derive the spatial correlation of two arbitrary channels between the source and the destination via relay stations in terms of the direction-delay spread functions of the links. The results show that the coherent components that exist in the channels between the source and the relay stations and in the channels between the destination and the relay stations lead to the non-zero cross-correlation among the links. Channel measurement results in two indoor environments are used to illustrate the existence of cross-correlation among the channels with the link ends located in different environments. These observations together with the theoretical work indicate that it is possible to use the geometry-based approaches that take into account the properties of the propagation environments to model the spatial correlation matrix of the multi-link MIMO channels.
\end{abstract}

Index Terms-Relay channel, MIMO, amplify-and-forward, channel characterization, channel modeling, wave propagation, spatial correlation, and spread function

\section{INTRODUCTION}

Wireless transmission technologies based on relay topologies, such as the multi-hop communication and cooperative/coordinated multi-point (CoMP) transmission techniques, are widely used in communication systems for throughput enhancement and coverage extension [1], [2]. Designing and optimizing the performance of these technologies require profound knowledge and insights into the multi-link propagation channels in different application scenarios. Recently, channel characterization and modeling for the relay channels have gained a lot of research attention [3], [4], [5], [6], [7], [8], [9].

Conventional studies on characterization and modeling of relay channels focus on the properties of individual links and their joint behavior, e.g. correlation. Relay channel measurements

This work was jointly supported by the IT R\&D program of MKE/KCC/KEIT. [KI002060, Wideband Wireless Channel Modeling based on IMT-Advanced], the project of the Science and Technology Commission of Shanghai Municipality [10ZR1432700, Multidimensional power spectrum characterization and modeling for wide-band propagation channels], the China Important National Science and Technology Specific Project [2009ZX03007-003-01, Research on Channel Measurement and Modeling for IMT-Advanced System], the China Education Ministry "New-teacher" Project [20090072120015, Time-Variant Channel Characterization, Parameter Estimation and Modeling] and the 985 program project of Tongji University [Wireless channel analysis and testing for the 4 th generation mobile communications]. are usually conducted by measuring multiple links sequentially using one channel sounde [10], [6], or measuring e.g. two links using two back-to-back configured sounders simultaneously [4]. The characteristics of individual links, such as the path loss, shadow fading and multipath fading, composite delay and angular spreads have been extensively investigated for the links between the source and relay, and between the relay and destination in both indoor and outdoor scenarios [5], [8], [10], [7]. However, the correlation models of multilink as specified in [10], [4], [6] are for calibration purposes with site-specific setup, and hence, cannot be applied for channel simulation in the performance assessment for communication systems.

Modeling the multi-link cross-correlation is nontrivial, considering the variable choices for the locations of the link ends, various types of the environments for the links, as well as different application contexts for the relay systems. Deterministic modeling based on ray-tracing techniques can only provide calibration models unsuitable for performance evaluation in a system level. The geometry-based approaches have been widely used for establishing statistical channel models, such as the 3GPP/3GPP2 spatial channel models (SCM) [11], and the WINNER enhanced SCM models [12]. Recently, using geometry-based approaches to model channel temporal auto-correlation has been proposed in [13]. Therefore, using similar geometry-based methods to model spatial cross-correlation of multi-links is also theoretically viable. However, since an amplify-and-forward (AF) relay channel can have a sophisticated spread function as described in [14], we need to consider a modeling scheme that is applicable for modeling the cross-correlation of multi-links using geometric approaches.

In this contribution, we first derive an analytical expression of the cross-correlation matrix for the multi-links in an $\mathrm{AF}$ relay system. Then we present a theoretical analysis of the mechanism for the cross-correlation by using the spread functions of multi-link propagation. Furthermore, we use channel measurement data collected in an office and an corridor indoor environments to evaluate the existence of non-zero crosscorrelation in reality.

The organization of the paper is as follows. In Section II the channel transfer matrix, correlation matrix for the multi-links in an AF-relay system is presented. In Section III, we derive the expression of the cross-correlation of two links in terms of spread functions. In Section IV, experimental investigations are conducted for checking the existence of cross-correlation 


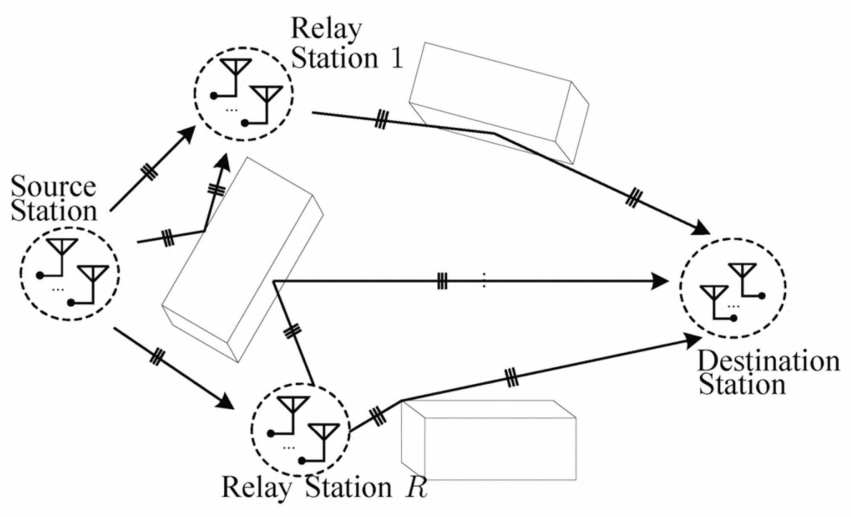

Fig. 1. Example of a propagation channel with multiple MIMO relay stations between a single source station and a single destination station

among links in indoor scenarios. Finally, conclusive remarks are made in Section V.

\section{Channel TRANSFER MATRIX AND CORRELATION MATRIX FOR AN AF RELAY SYSTEM}

We consider the case where the relay stations operate in the AF mode. This system consists of a source station, a destination station and $R$ relay stations. The source station and the destination station are equipped with respectively an $N$-element and $M$-element antenna array. The $r$ th relay station is equipped with $K_{r}$ antennas $(r=1, \ldots, R)$. Fig. 1 depicts an example of the relay system considered. The transfer matrix $H_{21} \in \mathcal{C}^{M \times N}$ of the propagation channel between the source station and destination station can be calculated as [15]

$$
\boldsymbol{H}_{21}=\boldsymbol{H}_{2} \boldsymbol{G} \boldsymbol{H}_{1}^{\mathrm{T}},
$$

where $(\cdot)^{\mathrm{T}}$ represents the matrix transposition, $\boldsymbol{H}_{1}$ and $\boldsymbol{H}_{2}$ denote respectively the transfer matrix between the source and the $R$ relay stations, and between the destination and the $R$ relay stations, and $G$ is the transfer matrix of the relay stations. The matrices $\boldsymbol{H}_{1}, \boldsymbol{H}_{2}$ and $\boldsymbol{G}$ can be written as

$$
\begin{aligned}
\boldsymbol{H}_{1} & =\left[\boldsymbol{H}_{1,1}, \ldots, \boldsymbol{H}_{1, r}, \ldots, \boldsymbol{H}_{1, R}\right] \in \mathcal{C}^{N \times K}, \\
\boldsymbol{H}_{2} & =\left[\boldsymbol{H}_{2,1}, \ldots, \boldsymbol{H}_{2, r}, \ldots, \boldsymbol{H}_{2, R}\right] \in \mathcal{C}^{M \times K}, \\
\boldsymbol{G} & =\operatorname{diag}\left(\boldsymbol{G}_{1}, \ldots, \boldsymbol{G}_{r}, \ldots, \boldsymbol{G}_{R}\right) \in \mathcal{C}^{K \times K},
\end{aligned}
$$

where $\boldsymbol{H}_{1, r} \in \mathcal{C}^{N \times K_{r}}$ represents the channel transfer matrix between the source and the $r$ th relay station, $\boldsymbol{H}_{2, r} \in \mathcal{C}^{M \times K_{r}}$ denotes the channel transfer matrix between the destination and the $r$ th relay station, $\boldsymbol{G}_{r} \in \mathcal{C}^{K_{r} \times K_{r}}$ is the channel transfer matrix between the $K_{r}$ receiving $(\mathrm{Rx})$ antennas and the $K_{r}$ transmitting (Tx) antennas, and $K=\sum_{r=1}^{R} K_{r}$. Without loss of generality, we consider the scenario where an AF relay station uses a certain amount of antennas to receive signals in one time-slot and transmits the amplified signals through the same antennas in the next time-slot. In such a case, $\boldsymbol{G}_{r}$ can be written as a diagonal matrix, i.e.

$$
\boldsymbol{G}_{r}=\operatorname{diag}\left(G_{r, 1}, \ldots, G_{r, k}, \ldots, G_{r, K_{r}}\right)
$$

with $G_{r, k}$ denoting the gain of the relay station.
Inserting (2)-(4) in (1) we obtain for $\boldsymbol{H}_{21}$

$$
\boldsymbol{H}_{21}=\sum_{r=1}^{R} \boldsymbol{H}_{2, r} \boldsymbol{G}_{r} \boldsymbol{H}_{1, r}^{\mathrm{T}} .
$$

The entries of $\boldsymbol{H}_{21}$ can be calculated as

$$
H_{21}[m, n]=\sum_{r=1}^{R} \sum_{k=1}^{K_{r}} H_{2, r, m, k} G_{r, k} H_{1, r, n, k},
$$

where $H_{1, r, n, k}$ denotes the (transfer) coefficient of the channel between the $n$th Tx antenna in the source and the $k$ th antenna in the $r$ th relay station, and $H_{2, r, m, k}$ represents the coefficient of the channel between the $m$ th $\mathrm{Rx}$ antenna in the destination and the $k$ th antenna in the $r$ th relay station. From (6) it can be seen that $H_{21}[m, n]$ is the superstition of the coefficients of all possible channels between the $n$th source antenna and the $m$ th destination antenna via relay stations. Using matrix notations, (6) is rewritten as

$$
\begin{gathered}
H_{21}[m, n]=\boldsymbol{h}_{2, m} \boldsymbol{G} \boldsymbol{h}_{1, n}^{\mathrm{T}} \text { with } \\
\boldsymbol{h}_{2, m}=\left[H_{2,1, m, 1}, H_{2,1, m, 2}, \ldots, H_{2, R, m, K_{R}}\right]^{\mathrm{T}} \text { and } \\
\boldsymbol{h}_{1, n}=\left[H_{1,1, n, 1}, H_{1,1, n, 2}, \ldots, H_{1, R, n, K_{R}}\right]^{\mathrm{T}} .
\end{gathered}
$$

The capacity of an AF relay system depends on the characteristics of the correlation matrix $\boldsymbol{C}$ of the vector $\boldsymbol{h}_{21}$ containing channel transfer coefficients of the propagation channel. Here, $\boldsymbol{h}_{21}=\operatorname{vec}\left[\boldsymbol{H}_{21}\right]$ and $\boldsymbol{C}=\mathrm{E}\left[\boldsymbol{h}_{21} \boldsymbol{h}_{21}^{\mathrm{H}}\right]$, with vec[.] denoting the matrix vectorization operation. The entry of $\boldsymbol{C}$, which is denoted with $C_{m n, m^{\prime} n^{\prime}}$, can be calculated as

$$
\begin{aligned}
C_{m n, m^{\prime} n^{\prime}} & \doteq \mathrm{E}\left[H_{21}[m, n] H_{21}\left[m^{\prime}, n^{\prime}\right]^{*}\right] \\
& =\operatorname{sum}\left[\mathrm{E}\left[\boldsymbol{C}_{2, m m^{\prime}} \odot \boldsymbol{C}_{1, n n^{\prime}}\right] \odot \boldsymbol{C}_{g}\right],
\end{aligned}
$$

where $(\cdot)^{*}$ denotes the complex conjugate, $\odot$ represents the element-wise matrix product, $\operatorname{sum}[\cdot]$ is the sum of all elements in the matrix given as argument, and

$$
\boldsymbol{C}_{2, m m^{\prime}}=\boldsymbol{h}_{2, m} \boldsymbol{h}_{2, m^{\prime}}^{\mathrm{H}}, \boldsymbol{C}_{1, n n^{\prime}}=\boldsymbol{h}_{1, n} \boldsymbol{h}_{1, n^{\prime}}^{\mathrm{H}}, \boldsymbol{C}_{\boldsymbol{g}}=\boldsymbol{g}^{\mathrm{H}}
$$

with $\boldsymbol{g}=\operatorname{vec}[\boldsymbol{G}]$. We can also rewrite $(8)$ as

$$
C_{m n, m^{\prime} n^{\prime}}=\operatorname{sum}\left[\mathrm{E}\left[\boldsymbol{h}_{m, n} \boldsymbol{h}_{m^{\prime}, n^{\prime}}^{\mathrm{H}}\right]\right],
$$

where $\boldsymbol{h}_{m, n}=\boldsymbol{h}_{2, m} \odot \boldsymbol{g} \odot \boldsymbol{h}_{1, n}$ is a vector containing the coefficients of all channels between the $n$th source antenna and the $m$ th destination antenna via individual relay stations. It can be seen from (9) that $C_{m n, m^{\prime} n^{\prime}}$ relies on the cross-correlation among the possible links between $n$th source antenna and the $m$ th destination antenna via relay stations. More specifically, it can be easily derived from (9) that $C_{m n, m^{\prime} n^{\prime}}$ is the sum of the element-wise correlations among four channel vectors, i.e. $\boldsymbol{h}_{2, m}, \boldsymbol{h}_{2, m^{\prime}}, \boldsymbol{h}_{1, n}$ and $\boldsymbol{h}_{1, n^{\prime}}$.

\section{CROSS-CORRELATION OF RELAY LINKS BASED ON PROPAGATION}

The derivations in Sect. II indicate that in order to calculate the correlation matrix for the multi-links of a relay channel, we need to compute first the correlation among the hops between the source and the relay stations, and between the destination 
and the relay stations. In this section, the analytical expression of the correlation of two relay links is derived in terms of the spread functions of the relay channels. The result obtained can help find the correlation mechanism of the relay channels from the perspective of wave propagation.

In the considered case where $R$ relay stations are present, the spread function of the relay channel can be written as

$$
h\left(\tau, \nu, \boldsymbol{\Omega}_{\mathrm{DoD}}^{\mathrm{S}}, \boldsymbol{\Omega}_{\mathrm{DoA}}^{\mathrm{D}}\right)=\sum_{r=1}^{R} h_{r}\left(\tau, \nu, \boldsymbol{\Omega}_{\mathrm{DoD}}^{\mathrm{S}}, \boldsymbol{\Omega}_{\mathrm{DoA}}^{\mathrm{D}}\right) .
$$

It has been derived in [16] that the spread function $h_{r}\left(\tau, \nu, \boldsymbol{\Omega}_{\mathrm{DoD}}^{\mathrm{S}}, \boldsymbol{\Omega}_{\mathrm{DoA}}^{\mathrm{D}}\right)$ of the propagation channel between the source and the destination via the $r$ th relay station can be calculated as

$$
\begin{gathered}
h_{r}\left(\tau, \nu, \boldsymbol{\Omega}_{\mathrm{DoD}}^{\mathrm{S}}, \boldsymbol{\Omega}_{\mathrm{DoA}}^{\mathrm{D}}\right)=\int_{\mathcal{D}} h_{r}\left(\tau, \nu, \boldsymbol{\Omega}_{\mathrm{DoD}}^{\mathrm{S}}, \boldsymbol{\Omega}_{\mathrm{DoA}}^{\mathrm{R}}, \boldsymbol{\Omega}_{\mathrm{DoD}}^{\mathrm{R}},\right. \\
\left.\boldsymbol{\Omega}_{\mathrm{DoA}}^{\mathrm{D}}\right) w_{r}\left(\boldsymbol{\Omega}_{\mathrm{DoA}}^{\mathrm{R}}, \boldsymbol{\Omega}_{\mathrm{DoD}}^{\mathrm{R}}\right) \mathrm{d} \boldsymbol{\Omega}_{\mathrm{DoA}}^{\mathrm{R}} \mathrm{d} \boldsymbol{\Omega}_{\mathrm{DoD}}^{\mathrm{R}},
\end{gathered}
$$

where $\mathcal{D}$ denotes the region where the integral is calculated, $\tau$ represents delay, $\nu$ denotes Doppler frequency, $\Omega_{\mathrm{DoD}}^{\mathrm{S}}$ is the direction of departure (DoD) in the source station, $\boldsymbol{\Omega}_{\mathrm{DoA}}^{\mathrm{R}}$ and $\Omega_{\mathrm{DoD}}^{\mathrm{R}}$ represent respectively the direction of arrival (DoA) and DoD at the $r$ th relay station, $\Omega_{\text {DoA }}^{\mathrm{D}}$ is the DoA in the destination station, and $\lambda$ denotes the wavelength. In an AF relay system, the conventional bi-direction-delay-Doppler frequency spread function of propagation can be extended to include two additional directions, i.e. DoA and DoD in the relay station. The amount of the channel parameters in the angular domains for a single path is now doubled comparing to the traditional generic path model [17]. The function $w_{r}\left(\boldsymbol{\Omega}_{\mathrm{DoA}}^{\mathrm{R}}, \boldsymbol{\Omega}_{\mathrm{DoD}}^{\mathrm{R}}\right)$ in (11) denotes the response of the $r$ th relay station

$$
w_{r}\left(\boldsymbol{\Omega}_{\mathrm{DoA}}^{\mathrm{R}}, \boldsymbol{\Omega}_{\mathrm{DoD}}^{\mathrm{R}}\right)=<\boldsymbol{c}^{\mathrm{Tx}}\left(\boldsymbol{\Omega}_{\mathrm{DoD}}^{\mathrm{R}}\right) \cdot \boldsymbol{c}^{\mathrm{Rx}}\left(\boldsymbol{\Omega}_{\mathrm{DoA}}^{\mathrm{R}}\right)>,
$$

where $c_{r}^{\mathrm{Tx}}\left(\Omega_{\mathrm{DoD}}^{\mathrm{R}}\right)$ and $c_{r}^{\mathrm{Rx}}\left(\Omega_{\mathrm{DoA}}^{\mathrm{R}}\right)$ are the responses of the Tx and $\mathrm{Rx}$ antenna arrays of the $r$ th relay station at the direction $\Omega_{\text {DoD }}^{\mathrm{R}}$ and direction $\Omega_{\text {DoA }}^{\mathrm{R}}$ respectively, and $\langle\cdot\rangle$ in the inner product of the vectors given as arguments.

It has been shown in [14] that

$$
\begin{aligned}
& h_{r}\left(\tau, \nu, \boldsymbol{\Omega}_{\mathrm{DoD}}^{\mathrm{S}}, \boldsymbol{\Omega}_{\mathrm{DoA}}^{\mathrm{R}}, \boldsymbol{\Omega}_{\mathrm{DoD}}^{\mathrm{R}}, \boldsymbol{\Omega}_{\mathrm{DoA}}^{\mathrm{D}}\right)= \\
& \quad h_{1, r}\left(\tau, \nu, \boldsymbol{\Omega}_{\mathrm{DoD}}^{\mathrm{S}}, \boldsymbol{\Omega}_{\mathrm{DoA}}^{\mathrm{R}}\right) * h_{2, r}\left(\tau, \nu, \boldsymbol{\Omega}_{\mathrm{DoD}}^{\mathrm{R}}, \boldsymbol{\Omega}_{\mathrm{DoA}}^{\mathrm{D}}\right),
\end{aligned}
$$

where $h_{1, r}\left(\tau, \nu, \mathbf{\Omega}_{\mathrm{DoD}}^{\mathrm{S}}, \mathbf{\Omega}_{\mathrm{DoA}}^{\mathrm{R}}\right)$ denotes the spread function of the channel between the source station and the $r$ th relay station, and $h_{2, r}\left(\tau, \nu, \Omega_{\mathrm{DoD}}^{\mathrm{R}}, \boldsymbol{\Omega}_{\mathrm{DoA}}^{\mathrm{D}}\right)$ represents the spread function of the channel between the $r$ th relay station and the destination station.

It is straightforward to show that the entries of the channel correlation matrix $C$ can be calculated by applying Fourier transformation to the auto-correlation function of the relay channel in delay, Doppler frequency, and bi-directions, which we denote with $\zeta\left(\tau, \nu, \boldsymbol{\Omega}_{\mathrm{DoD}}^{\mathrm{S}}, \boldsymbol{\Omega}_{\mathrm{DoA}}^{\mathrm{D}}\right)$. This function can be calculated as

$$
\begin{aligned}
& \zeta\left(\tau, \nu, \boldsymbol{\Omega}_{\mathrm{DoD}}^{\mathrm{S}}, \boldsymbol{\Omega}_{\mathrm{DoA}}^{\mathrm{D}}\right) \\
& \quad \doteq \mathrm{E}\left[h\left(\tau, \nu, \boldsymbol{\Omega}_{\mathrm{DoD}}^{\mathrm{S}}, \boldsymbol{\Omega}_{\mathrm{DoA}}^{\mathrm{D}}\right) h^{*}\left(\tau, \nu, \boldsymbol{\Omega}_{\mathrm{DoD}}^{\mathrm{S}}, \boldsymbol{\Omega}_{\mathrm{DoA}}^{\mathrm{D}}\right)\right] \\
& \quad=\sum_{r=1}^{R} \sum_{r^{\prime}=1}^{R} \zeta_{r r^{\prime}}\left(\tau, \nu, \boldsymbol{\Omega}_{\mathrm{DoD}}^{\mathrm{S}}, \boldsymbol{\Omega}_{\mathrm{DoA}}^{\mathrm{D}}\right)
\end{aligned}
$$

where $\zeta_{r r^{\prime}}\left(\tau, \nu, \boldsymbol{\Omega}_{\mathrm{DoD}}^{\mathrm{S}}, \boldsymbol{\Omega}_{\mathrm{DoA}}^{\mathrm{D}}\right)$ is the cross-correlation function between the propagation channels with the $r$ th relay station and the $r^{\prime}$ th relay station, i.e.

$$
\begin{aligned}
& \zeta_{r r^{\prime}}\left(\tau, \nu, \boldsymbol{\Omega}_{\mathrm{DoD}}^{\mathrm{S}}, \boldsymbol{\Omega}_{\mathrm{DoA}}^{\mathrm{D}}\right) \\
& =\mathrm{E}\left[h_{r}\left(\tau, \nu, \boldsymbol{\Omega}_{\mathrm{DoD}}^{\mathrm{S}}, \boldsymbol{\Omega}_{\mathrm{DoA}}^{\mathrm{D}}\right) h_{r^{\prime}}^{*}\left(\tau, \nu, \boldsymbol{\Omega}_{\mathrm{DoD}}^{\mathrm{S}}, \boldsymbol{\Omega}_{\mathrm{DoA}}^{\mathrm{D}}\right)\right] .
\end{aligned}
$$

The assumption that $h_{r}\left(\tau, \nu, \boldsymbol{\Omega}_{\mathrm{DoD}}^{\mathrm{S}}, \boldsymbol{\Omega}_{\mathrm{DoA}}^{\mathrm{D}}\right), r=1, \ldots, R$ are stochastic orthogonal measures has been used in [14] for simplifying the computation of (14). However, this assumption may not hold in reality. Experimental investigations reported in Sect. IV show that the cross-correlation of different channels can be significant, depending on the propagation environments. In order to find the correlation mechanism, we assume that the antennas of the relay stations are isotropic, i.e. $w_{r}\left(\boldsymbol{\Omega}_{\text {DoA }}^{\mathrm{R}}, \boldsymbol{\Omega}_{\mathrm{DoD}}^{\mathrm{R}}\right)=1$. In such a case, the cross-correlation of spread functions can be calculated as

$$
\begin{aligned}
& \zeta_{r r^{\prime}}\left(\tau, \nu, \boldsymbol{\Omega}_{\mathrm{DoD}}^{\mathrm{S}}, \boldsymbol{\Omega}_{\mathrm{DoA}}^{\mathrm{D}}\right) \\
& =\mathrm{E}\left[\left(h_{1, r}\left(\tau, \nu, \boldsymbol{\Omega}_{\mathrm{DoD}}\right) * h_{2, r}\left(\tau, \nu, \boldsymbol{\Omega}_{\mathrm{DoA}}\right)\right)\right. \\
& \left.\quad \cdot\left(h_{1, r^{\prime}}^{*}\left(\tau, \nu, \boldsymbol{\Omega}_{\mathrm{DoD}}\right) * h_{2, r^{\prime}}^{*}\left(\tau, \nu, \boldsymbol{\Omega}_{\mathrm{DoA}}\right)\right)\right] \\
& =\mathrm{E}\left[h_{1, r, r^{\prime}}\left(\tau, \nu, \boldsymbol{\Omega}_{\mathrm{DoD}}\right) * h_{2, r, r^{\prime}}\left(\tau, \nu, \boldsymbol{\Omega}_{\mathrm{DoA}}\right)\right],
\end{aligned}
$$

where

$h_{1, r, r^{\prime}}\left(\tau, \nu, \boldsymbol{\Omega}_{\mathrm{DoD}}\right)=h_{1, r}\left(\tau, \nu, \boldsymbol{\Omega}_{\mathrm{DoD}}\right) h_{1, r^{\prime}}\left(\tau, \nu, \boldsymbol{\Omega}_{\mathrm{DoD}}\right)$

is the joint spread function of the links between the source and the $r$ th relay station and between the soruce and the $r^{\prime}$ th relay station, and

$h_{2, r, r^{\prime}}\left(\tau, \nu, \boldsymbol{\Omega}_{\mathrm{DoA}}\right)=h_{2, r}\left(\tau, \nu, \boldsymbol{\Omega}_{\mathrm{DoA}}\right) h_{2, r^{\prime}}\left(\tau, \nu, \boldsymbol{\Omega}_{\mathrm{DoA}}\right)$

is the joint spread function of the links between the destination station and the $r$ th relay station, and between the destination station and the $r^{\prime}$ th relay station.

From (16), (17) and (18) it is evident that the links in the relay system considered are correlated if and only if the following conditions hold: $i$ ) the spread functions of the links between the source and the relay stations have common components; ii) the spread functions of the links between the destination and the relay stations have common parts; iii) these common parts are correlated. The conditions $i$ ) and ii) hold in the case where the constellations of the propagation paths exhibit certain degree of similarity for the channels. The condition iii) holds when the common components identified from the former two conditions are coherent. This implies that the common components have to be the line-of-sight (LOS) components or the dominant components characterized with Rician fading. 


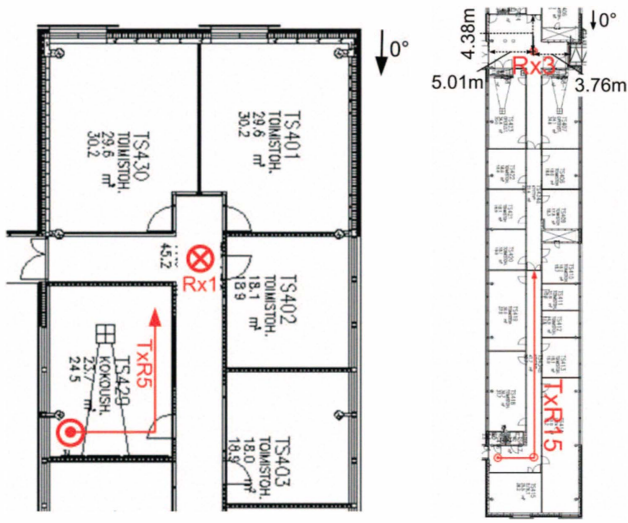

Fig. 2. The maps of the environment and the measurement settings for the office scenario (a) and the corridor scenario (b)

\section{EXPERIMENTAL INVESTIGATION OF CROSS-CORRELATION AMONG LINKS}

In this section, channel measurement data are used to check the cross-correlation of the channels in indoor environments. The observations can help understand the relationship between the cross-correlation and the propagation environments.

The measurements were organized by Technology University of Vienna and Elektrobit in Oulu University, using the wideband MIMO channel sounder - Propsound [18]. For the detail description of the sounder and the measurement campaign, readers are referred to [19]. The Tx and $\mathrm{Rx}$ were equipped with antenna array of 50 and 32 elements respectively. The $\mathrm{Rx}$ was fixed in the measurements. The Tx was moving along specific routes at speed about $0.5 \mathrm{~m} / \mathrm{s}$. The bandwidth of the sounder equals $100 \mathrm{MHz}$. The carrier frequency was 5.25 $\mathrm{GHz}$. Channels between all $\mathrm{Tx}$ antennas and $\mathrm{Rx}$ antennas are measured sequentially in time-division-multiplexing (TDM) mode. In each measurement cycle, $50 \times 32=1600$ subchannels are measured once. The data acquisition is activated for the consecutive four cycles, which we call as "burst". Then after the duration of 12 cycles, the data acquisition is activated again. The movement of the Tx in cycle is small in such a way that the channel can be assumed invariant within the period of one cycle. Since the antenna radiation patterns are not exactly the same for the $\mathrm{Tx}$ and $\mathrm{Rx}$ antennas, and in addition, the system responses have random phase noise when measuring different subchannels, we can consider the channel impulse responses obtained from the 1600 subchannels as independent observations of the wide-sense-stationary channel. The narrowband channel coefficients are calculated by summing up the impulse responses of individual subchannels. The crosscorrelation coefficient $\rho_{x^{\prime} x^{\prime \prime}}$ of the channels with coefficients $x^{\prime}$ and $x^{\prime \prime}$ are calculated as

$$
\rho_{x^{\prime} x^{\prime \prime}}=\left|\mathrm{E}\left[x^{\prime} x^{\prime \prime *}\right]-\mu_{x^{\prime}} \mu_{x^{\prime \prime}}\right| /\left(\sigma_{x^{\prime}} \sigma_{x^{\prime \prime}}\right),
$$

where $\mu_{x}$ and $\sigma_{x}$ denote respectively the mean and standard deviation of the process $x$.

The measurement data obtained in an office and a corridor

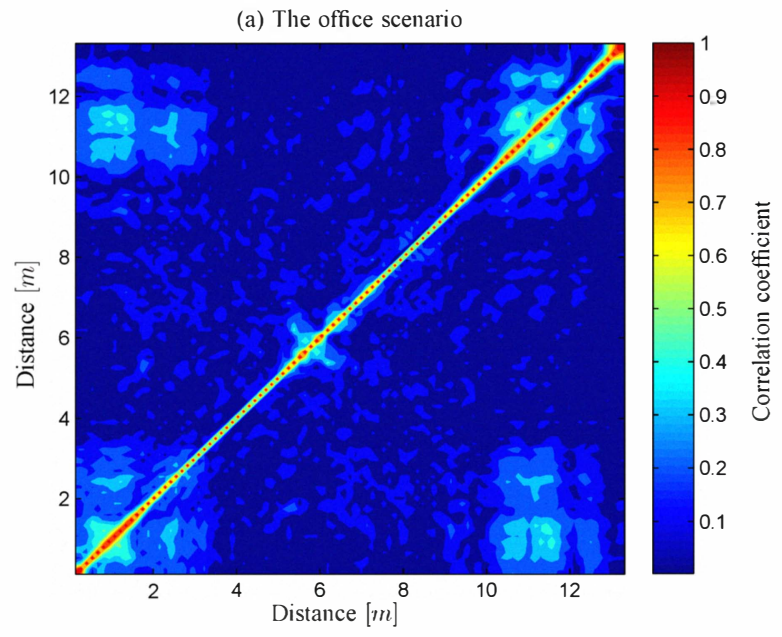

(b) The corridor scenario

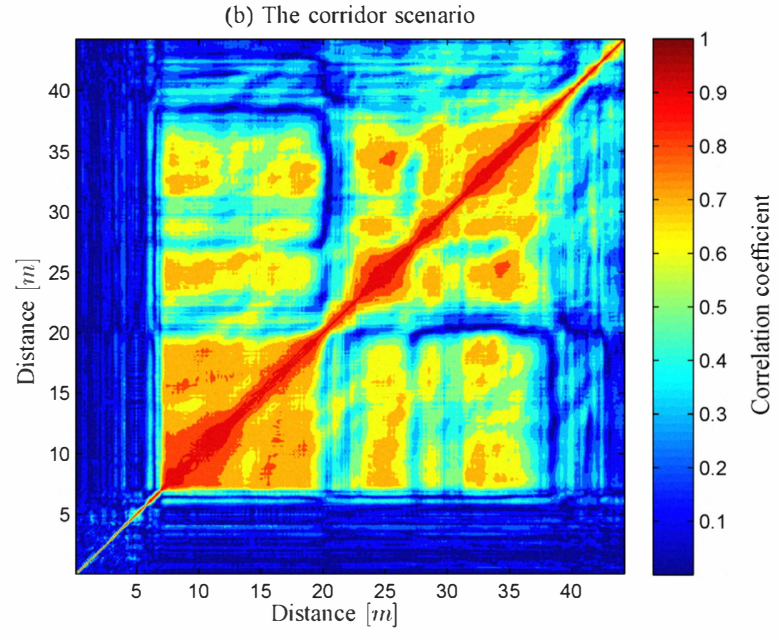

Fig. 3. The contour plots of the spatial cross-correlation matrix of the channels observed along the measurement routes for the office scenario (a) and the corridor scenario (b).

scenarios are used for evaluating the spatial correlation of the links. In the former scenario, the measurement was performed with the Tx moving in an office. In the latter scenario, the Tx moved in a narrow corridor with offices on both sides. The measurements in the narrow corridor can be divided in two parts, the non-line-of-sight (NLOS) and the line-ofsight (LOS) scenarios. The maps of the environments for the scenarios considered are shown in Fig. 2, where the Tx started moving from the locations marked with a solid red spot, and stopped at the location marked with an arrow.

Fig. 3 (a) and (b) show the contour plot of the channel spatial correlation matrix for the office and corridor scenarios respectively. The $x-$ and $y$-axes represent the distance of the Tx from the starting point. We observe that the channel in office has much narrower spatial autocorrelation function than in the corridor scenario. Furthermore, we observe from Fig. 3 (b) that the channels before and after 6 meters from the starting point are quite different. This is reasonable because the propagation scenario changes from the NLOS case to the LOS case when the Tx moved around the corner of the corridor as shown in Fig. 2 (b). 
From the correlation matrices shown in Fig. 3 we observe that some channels measured with Tx locations far apart from each other are highly correlated. In Fig. 3 (a), the channels observed when the Tx moves in the range $[1,3]$ meters are correlated with the channels observed when the Tx moves in $[10,12]$ meters. We can see from the map shown in Fig. 2 (a) that for these two ranges, the Tx was located at two corners of the office, i.e. the locations close to image positions with respect to the door of the office. Significant cross-correlation can also be observed in Fig. 3 (b) for the corridor scenario. The channels observed when the Tx moved within the range $[7,30]$ meters are strongly correlated with the channels observed when the Tx moved in the range of $[20,37]$ meters. According to the map shown in Fig. 2 (b), when the Tx moved in the corridor within these ranges, the environments are very similar with each other. Our conjunction is that similar propagation environments lead to significant overlapping of the spread functions of the links. Furthermore, due to the wave-guiding effect of the corridor, the components are nearly coherent. These effects lead to the strong cross-correlation between the channels when the Tx moves within these two areas.

\section{CONCLUSIONS}

In this contribution, an analytical expression has been derived for the cross-correlation matrix of the relay channel which contains multiple links between the source and the relay stations, and between the destination and the relay stations. We also showed by theoretical derivation that the cross-correlation among the multiple links is actually determined by the joint characteristics of the spread functions of the propagation channels. More specifically, we found that the coherent components existing in the propagations between the source and the relay stations and between the destination station and the relay stations lead to the non-zero cross-correlations among links. Experimental results based on the measurements in two indoor scenarios have shown that when the transmitters or receivers of the two links are in image positions, or when the propagation environments are similar, strong cross-correlation can be observed. This effect is regardless of the distance between the locations of the link ends. The experimental observations and the theoretical results presented in the paper can help us find geometry-based approaches for constructing the correlation models for multi-link channels.

\section{ACKNOWLEDGEMENT}

The author wishes to thank Elektrobit Finland for kindly providing the measurement data.

\section{REFERENCES}

[1] H. Dai, H. Zhang, and Q. Zhou, "Some analysis in distributed MIMO systems," Journal of Communications, vol. 2, no. 3, pp. 43-50, May 2007.

[2] IEEE 802.16's Relay Task Group: IEEE 802.16j proposals., IEEE Std., 2009.
[3] A. Wittneben and B. Rankov, "Impact of cooperative relays on the capacity of rank-deficient MIMO channels," in Proceedings of the 12th IST Summit on Mobile and Wireless Communications, June 2003, pp. 421-425.

[4] J. Koivunen, P. Almers, V.-M. Kolmonen, J. Salmi, A. Richter, F. Tufvesson, P. Suvikunnas, A. Molisch, and P. Vainikainen, "Dynamic multi-link indoor MIMO measurements at $5.3 \mathrm{GHz}$," Proceedings of the second European conference on Antennas and Propagation, 2007, pp. 1-6, Nov. 2007.

[5] N. Czink, B. Bandemer, and G. V. Vilar, "Spatial separation of multiuser MIMO channels," in COST 2100 TD (08) 622, Lille, Oct 2008.

[6] L. Jiang, L. Thiele, and V. Jungnickel, "Modeling and measurement of MIMO relay channels," in Proceedings of the Vehicular Technology Conference (VTC Spring), May 2008, pp. 419-423.

[7] J. Zhang, D. Dong, X. Nie, G. Liu, and W. Dong, "Propagation characteristics of wideband relay channels in urban environment," in Proceedings of The 3rd International Workshop on Broadband MIMO Channel Measurement and Modeling, August 25, 2009.

[8] X. Hu, Y. Zhang, Y. Jia, S. Zhou, and L. Xiao, "Power coverage and fading characteristics of indoor distributed antenna systems," in The 3rd International Workshop on Broadband MIMO Channel Measurement and Modeling (IWONCMM'09), August 2009.

[9] X. Yin, Y. Zhou, and F. Liu, "A generic wideband channel model for keyhole propagation scenarios and experimental evaluation," in Fourth International Conference on Communications and Networking in China, 2009. ChinaCOM 2009., Aug. 2009, pp. 1-5.

[10] C. Oestges, N. Czink, B. Bandemer, P. Castiglione, F. Kaltenberger, and A. Paulraj, "Experimental characterization and modeling of outdoor-toindoor and indoor-to-indoor distributed channels," Vehicular Technology, IEEE Transactions on, vol. PP, no. 99, pp. 1-1, 2010.

[11] Spatial channel model for Multiple Input Multiple Output (MIMO) simulations (Release 7), 3GPP TR25.996 V7.0.0 Std., 2007.

[12] WINNER II Channel Models, IST-WINNER II Deliverable 1.1.2 v.1.2. Std., IST-WINNER2. Tech. Rep., 2007. [Online]. Available: http://www.ist-winner.corg/deliverables.html996

[13] X. Yin, J. Zhou, B.-J. Kwak, and H. K. Chung, "Estimation of channel temporal auto-correlation and coherence time based on propagation paths," in Proceedings of the Fifth International Conference on Communications and Networking in China, 2010. ChinaCOM 2010., Sept. 2010, pp. 1-5.

[14] X. Yin and S. X. Lu, "Preliminary studies on stochastic modeling of propagation channels in multi-relay systems," in Submitted to IEEE vehicular Technology conference, VTC-Fall, 2010.

[15] M. Herdin, "MIMO amplify-and-forward relaying in correlated MIMO channels," in Proceedings of the Fifth International Conference on Information, Communications and Signal Processing, 2005, pp. 796800 .

[16] X. Yin, S. X. Lu, B.-J. Kwak, H. K. Chung, and F. Liu, "Propagation channel characterization for amplify-and-forward mimo-relaying systems," in Proceedings of IEEE the 72nd Vehicular Technology Conference, 2010VTC-Fall, 2010.

[17] A. Molisch, "A generic model for MIMO wireless propagation channels in macro- and microcells," IEEE Transactions on Signal Processing, vol. 52(1), pp. 61-71, Jan. 2004.

[18] P. Kyösti, J. Meinilä, L. Hentilä, X. Zhao, T. Jämsä, C. Schneider, M. Narandzić, M. Milojević, A. Hong, J. Ylitalo, V.-M. Holappa, M. Alatossava, R. Bultitude, Y. de Jong, and T. Rautiainen, "WINNER II Channel Models D1.1.2 V1.1," no. IST-4-027756 WINNER II, D1.1.2 V1.1, 112007

[19] N. Czink, "The random-cluster model - a stochastic mimo channel model for broadband wireless communication systems of the 3rd generation and beyond," Ph.D. dissertation, Technology University of Vienna, Department of Electronics and Information Technologies, December 2007. 